\title{
Isothermal kinetics Model for Solid-Solid Reaction through Surface Area and Size Distribution of the particle
}

\author{
Mingrui Yang ${ }^{1}$, Xuewei Lv², Chenguang Bai², and Hanyuan Wang ${ }^{1}$ \\ ${ }^{1}$ Affiliation not available \\ ${ }^{2}$ Chongqing University
}

October 18, 2020

\begin{abstract}
Many industrial processes such as metallurgical, chemistry, ceramic and cement industry often involve solid-solid reactions. To develop a quantitative representation of the solid-solid reaction of powders for the binary reactants system, the investigation of modelling which involved particle shape, surface area, particle size distribution and diffusion rate of reactants was carried out. In this model, the interface of particles were divided into reaction surface and non-reaction surface, and the particle shape was divided into the first level shape and the second level shape. The area of reaction interface was calculated, while the concentration distributions of reactants are numerically expressed. The simulated kinetics curves of fractional conversion $\alpha$ with time in different average particle size and in different diffusion coefficient were shown to be useful to evaluate the kinetics of solid-solid reactions. The simulated kinetics curves and experimental data from references were compared and it achieved good accuracy.
\end{abstract}

\section{Hosted file}

Manuscript.pdf available at https://authorea.com/users/368192/articles/487413-isothermalkinetics-model-for-solid-solid-reaction-through-surface-area-and-size-distribution-ofthe-particle

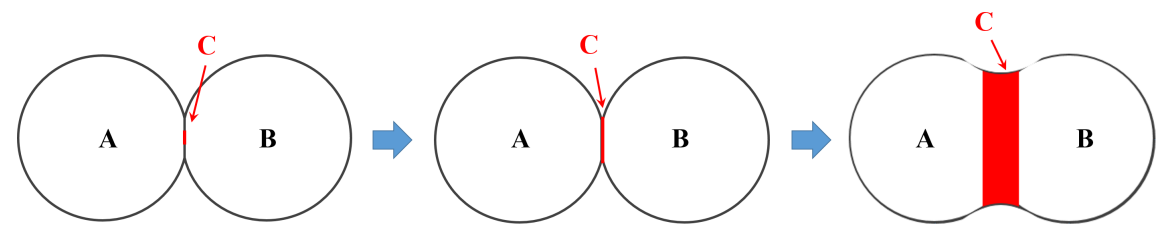



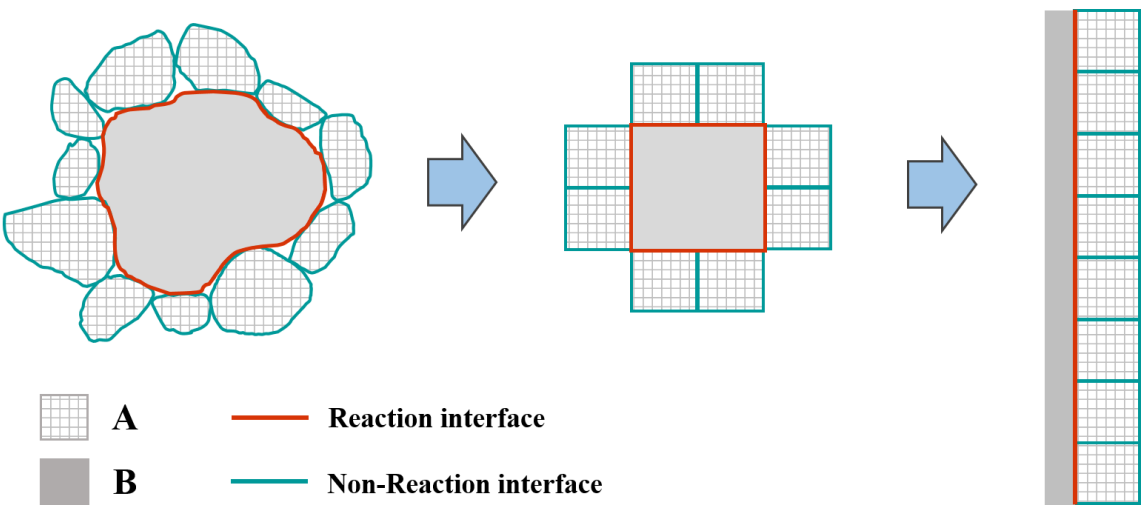
A
Reaction interface
B
Non-Reaction interface

(a)

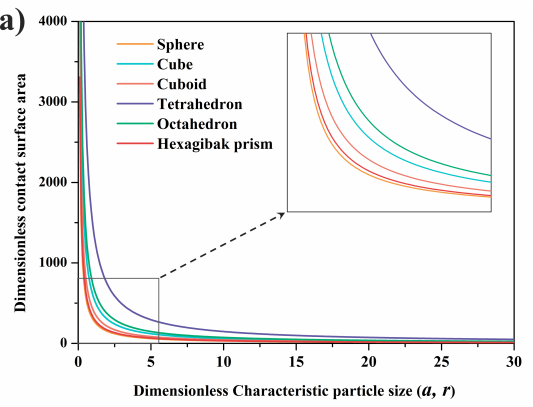

(a)

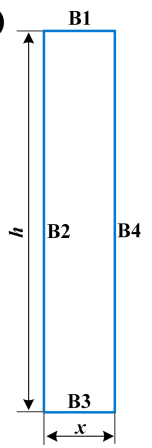

Boundary Conditions:

B1 no mass flux , no slip

B2 inflow boundary, no slip

B3 no mass flux, no slip

B4 no mass flux, no slip

Initial Conditions:

$C=C^{*}$ at $\mathbf{B} 2, t=0$

$C=0$ at B1 B3 B4, $t=0$

Control function:

$\frac{\partial C_{i}}{\partial t}+\nabla \cdot\left(-D_{i} \nabla C_{i}\right)=R_{i}$

$-\mathbf{n} \cdot \mathbf{N}_{i}=0$ (b)

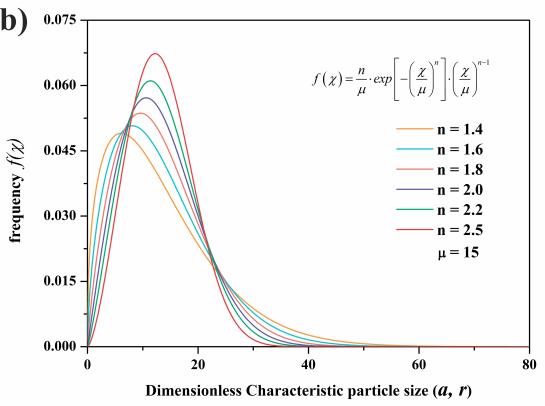

(b)

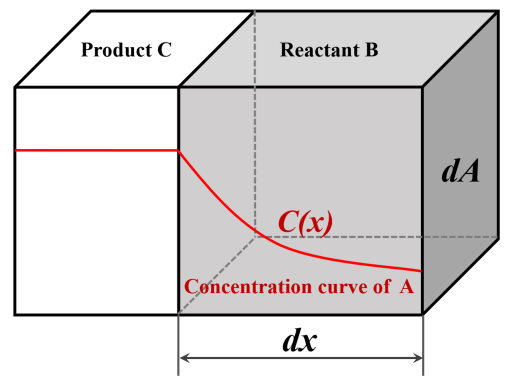




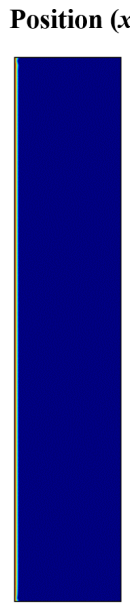

0

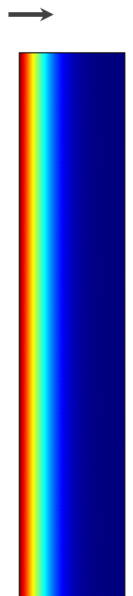

1200

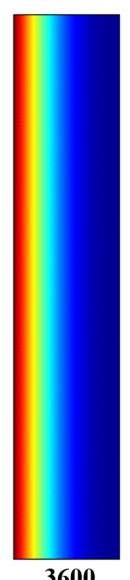

3600

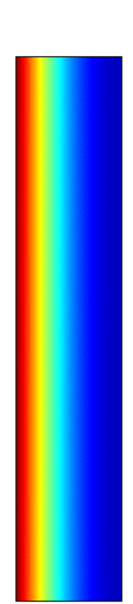

4800

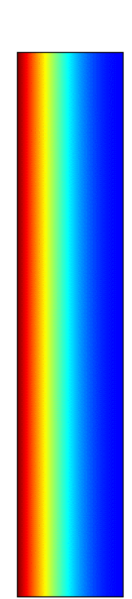

6400
Concentration ( $C$ )
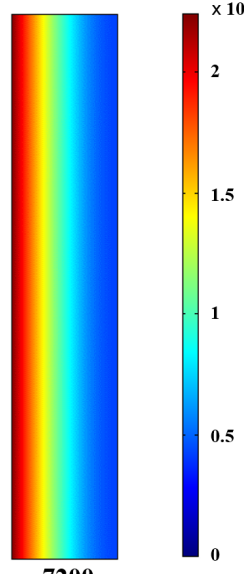

2

1.5

0.5

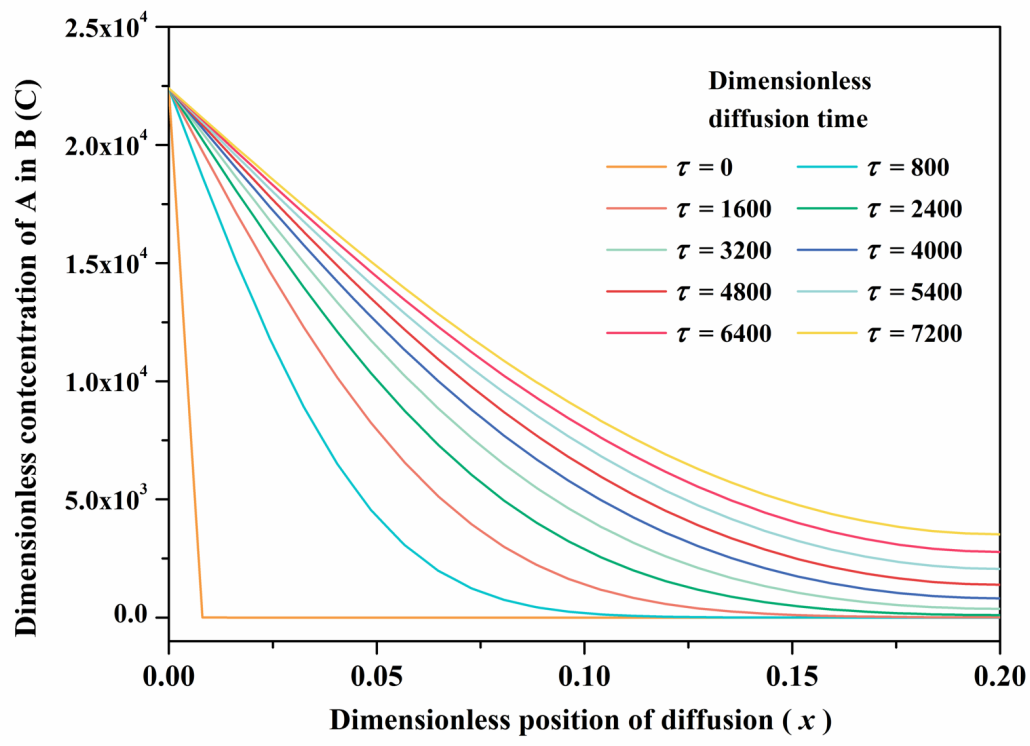



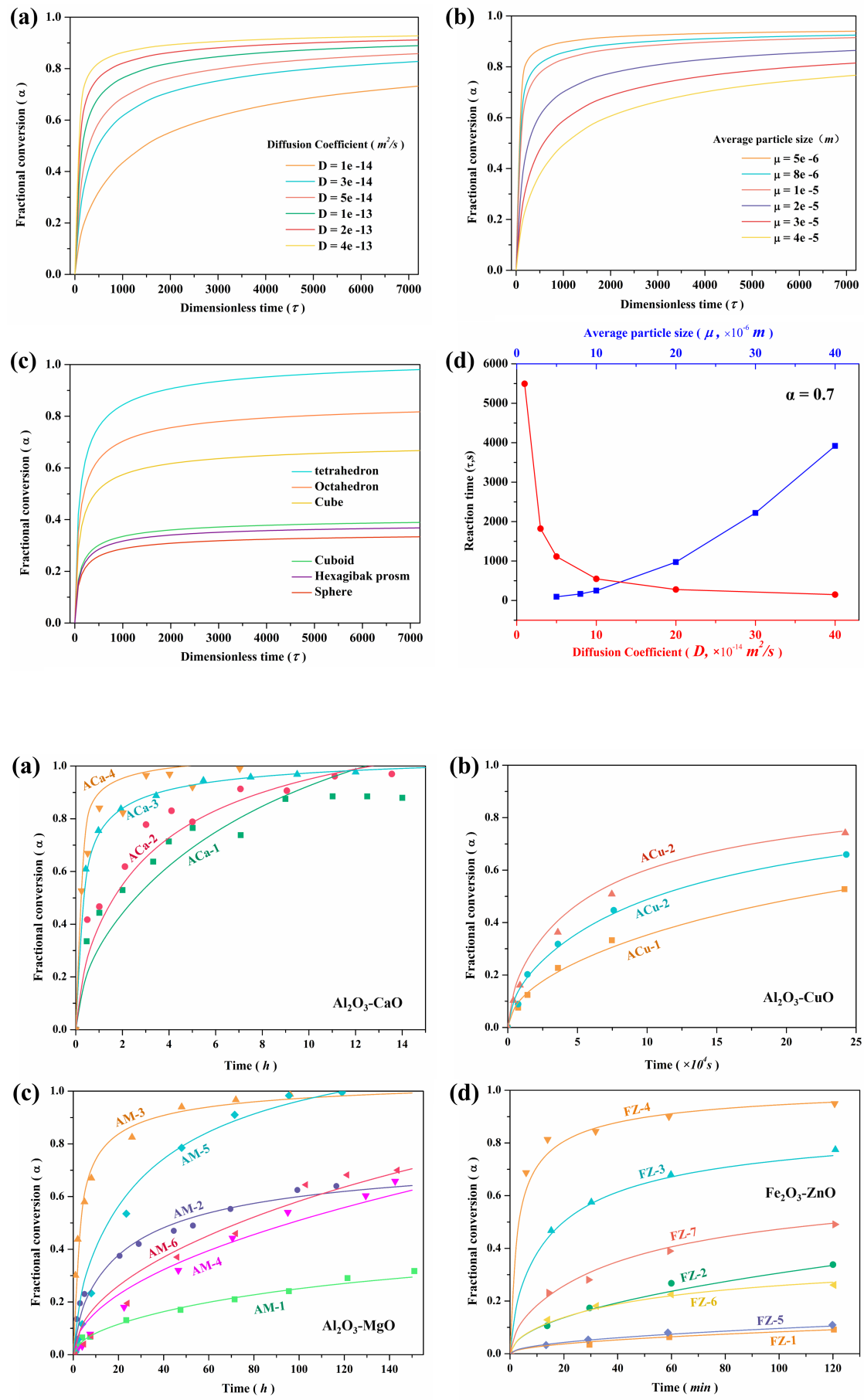

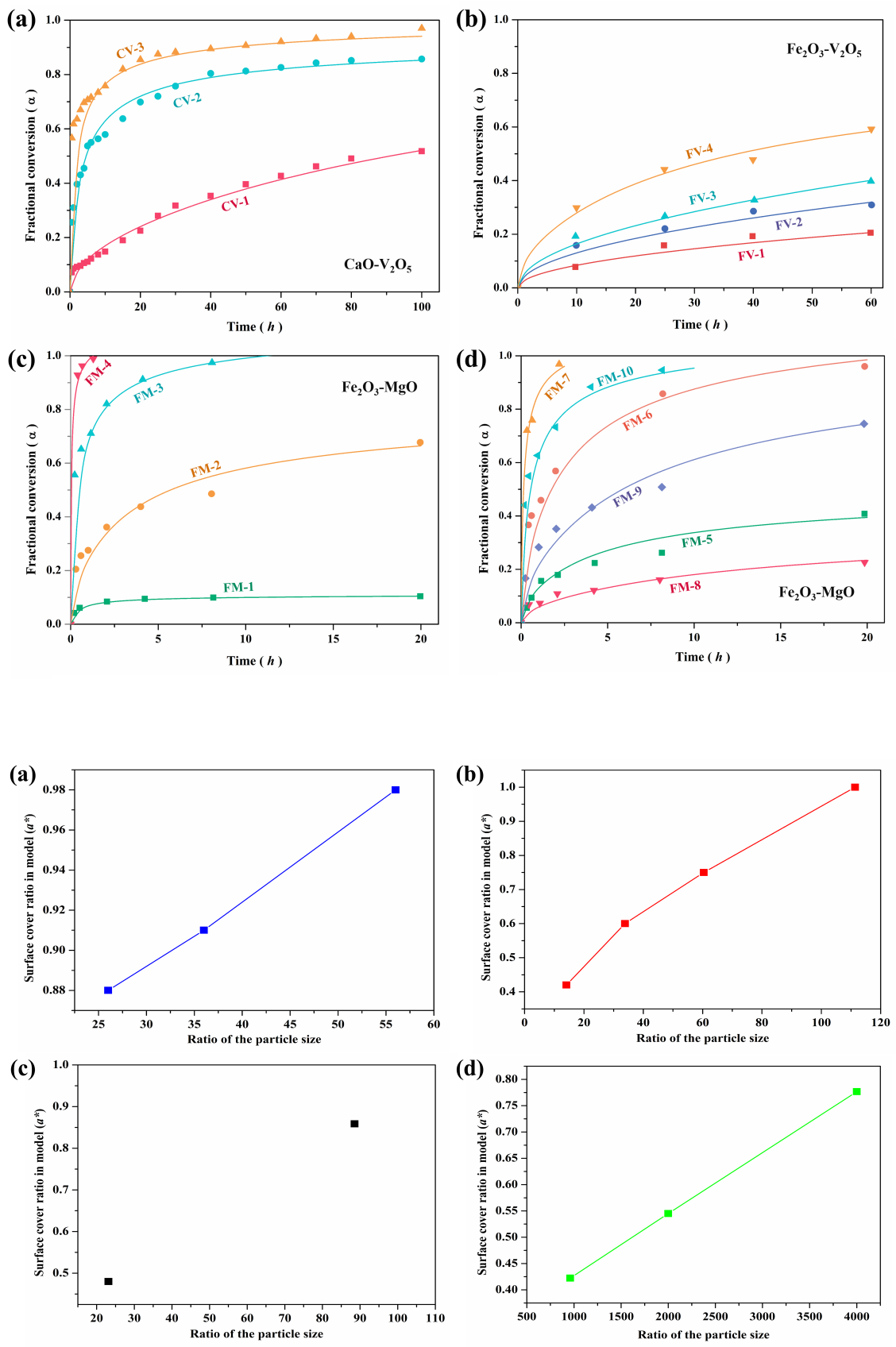